\title{
Achieving Efficient Decision Making Through Hybrid Reduction in Soft Set Theory
}

\author{
Ahmad Nazari Mohd Rose*, Mohd Isa Awang ${ }^{\#}$, Fadhilah Ahmad", Nurnadiah Zamri ${ }^{\#}$, Mohamad Afendee \\ Mohamed $^{\#}$, Mustafa Mat Deris \\ *Faculty of Informatics and Computing, Universiti Sultan Zainal Abidin, Besut, Terengganu, Malaysia \\ E-mail:anm@unisza.edu.my,isa@unisza.edu.my,fad@unisza.edu.my,nadiahzamri@unisza.edu.my,mafendee@unisza.edu,my \\ ${ }^{\#}$ Faculty of Computer Science and Information Technology, University Tun Hussein Onn Malaysia, Batu Pahat, Johor, Malaysia \\ E-mail: mmustafa@uthm.edu.my
}

\begin{abstract}
The main intention of proposing an alternative technique is to ensure consistency is been upheld besides successfully reducing the file. Of all the reduction techniques available currently, only normal parameter reduction has managed to address the issue of consistency at optimal and suboptimal level. In this paper, we initiated another form of reduction known as hybrid reduction by complementing the normal parameter reduction with object reduction. It has already demonstrated that the proposed hybrid reduction has successfully reduced data by $55 \%$ with the sample used, thus proving that it as a good alternative for the process of decision making using less amount of data.
\end{abstract}

Keywords - soft set theory; hybrid reduction; parameter reduction; normal reduction; object reduction; decision making

\section{INTRODUCTION}

Nowadays, the soft set theory as one of the mathematical principles has been applied in solving problems related to issues of uncertain data the soft set theory [1]. The soft set theory was initiated by Molodtsov in 1999.

In past years, the soft set theory has been actively studied. It has included researches in the fields of the fundamentals of soft set theory, soft set theory in abstract algebra and soft set theory for data analysis. The study of data reduction is vital, especially in the field of decision making [1]-[11]. Besides that, numerous researches have been developed rapidly in meeting the numerous demands in real-world situations especially with regards to soft set applications [12]-[14], data mining [15], [16] and so forth.

The soft set theory also has an impact in the field of data reduction [17]-[19] because the importance of the data reduction in helping to improve decision making with less amount of data. If a huge amount of information are involved during the process of decision making, then factors such as processing times and inefficiencies will be the main issues. Therefore, opting for a lesser amount of data to be processed will be a better alternative.

In order to perform reduction, one may encounter this problem. For a particular property, whether all the attributes in the set of attributes are always necessary to conserve this property [20]. The need of sophisticated examination methods to illuminate features, which would be readily apparent in small datasets [21]. Besides that, we also need to ensure that the data in reduced form is still considered as quality data [22].

The main motivation of our study is to present a new approach to reduction based on soft set theory. As most of the contribution to data reduction in soft set theory are based on parameter reduction, object reduction has never been discussed. There are times when objects that exist in the dataset are of a non-quality material, but cannot be ignored as in [2]-[4]. This issue has been tackled in this study by eliminating or partitioning certain columns or rows that adhere to our definition of dispensable objects and parameters.

Application of soft sets in a decision-making problem with the help of rough sets is presented [1]. But the main problem is that different proposed dispensable dataset will output different optimal object, which affects the issue of the consistency in decision making. However, the issue of inconsistency has addressed but has only succeeded in achieving consistency at the optimal object level [3]. Although able to maintain consistency, but it has not been able to reduce data substantially [4]. In our paper, supported sets are used to derive maximal support. The obtained maximal supported set will be instrumental in our proposed reduction whereby column reduction will be complemented by row reduction. We have also proven that the proposed 
hybrid reduction performs better in terms of data size and consistency.

The rest of this paper is organized as follows. Section I describes the concept of reduction in rough set theory. Section I also describes the fundamental concept of soft set theory. Section II presents the analysis of previous works on data reduction and decision making in the soft set theory. An alternative technique of data reduction and decision making based on hybrid reduction using supported sets is introduced in section III. And we conclude our works in section IV.

\section{A. Rough Set Theory}

The rough sets are defined through their dual set approximations in an information system. A pair of dual set approximations is used to approximate rough subset of the set of objects is called a lower and an upper approximation in term of these equivalence classes [23].

\section{B. Information System}

An information system [11] is a 4-tuple (quadruple), $S=(U, A, V, f)$ where $U$ is a non-empty finite set of objects, $A$ is a non-empty finite set of attributes, $V=\bigcup_{a \in A} V_{a}$, $V_{a}$ is the domain (value set) of attribute $a, f: U \times A \rightarrow V$ is a total function such that $f(u, a) \in V_{a}$, for every $(u, a) \in U \times A$ is identified as information (knowledge) function.

Every non-empty subset of $A$ induces unique indiscernibility relation denoted by $\operatorname{IND}(B)$ that is induced by the set of attribute $B$, is an equivalence relation that eventually induces a unique partition. The induced partition of $U, I N D(B)$ in $S=(U, A, V, f)$ is denoted by $U / B$ and the equivalence class in the partition $U / B$ consisting of $x \in U$, denoted by $[x]_{B}$. Thus, $\operatorname{IND}(B)=\bigcap_{a \in B} \operatorname{IND}\{a\}$ is obtained.

\section{Reducts and Core}

A reduct is identified as the minimal set of attributes that uphold the indiscernibility relation. A core is the most common parts of all of the identified reducts that is presented by the following preliminaries definitions.

Definition 1: (See [2]) Let $S=(U, A, V, f)$ be an information system and let $B$ be any subsets of $A$. If all its attributes are indispensable, then $B$ is called independent (orthogonal) set.

The above definition states that for any set of parameters that were not dispensable, it should never be considered at all in the process of reduction. If this is not heed, then the produced analytical results are no longer reliable to be used in the process of decision-making.

Definition 2: (See [2]) Let $S=(U, A, V, f)$ and let $B$ be any subsets of $A$. A subset $B *$ of $B$ is a reduct of $B$ if $B *$ is independent and $U / B^{*}=U / B$.
Thus, for any set of attributes that preserves partition is known as the reducts. One of the properties of reducts is known as core.

Definition 3: (See [2]) Let $S=(U, A, V, f)$ be an information system and let $B$ be any subsets of $A$. The core of $B$ is the intersection off all reducts.

$$
\operatorname{Core}(B)=\bigcap \operatorname{Red}(B)
$$

Since the core is the intersection of all reducts, it is thus found in every reduct. Thus, the core of $B$ is the set off all indispensable attributes of $B$.

\section{Soft Set Theory}

Throughout this section, $U$ is referred as the initial universe, $E$ is a set of parameters and $P(U)$ is the power set of $U$.

Definition 4: (See [2]) A pair $(F, E)$ is called a soft set over $U$, where $F$ is a mapping given by $F: E \rightarrow P(U)$

A soft set, in other words, is the parameterized family of subsets of the universe $U$. For $\varepsilon \in E, F(\varepsilon)$ can be regarded as the set of $\varepsilon$-elements of the soft set $(F, E)$. It can also be considered as the set of $\mathcal{E}$-approximate elements of the soft set, instead of a (crisp) set.

Definition 5: If $(F, E)$ is a soft set over the universe $U$, then if $S=\left(U, A, V_{\{0,1\}}, f\right)$, thus $(F, E)$ is the binary-valued information system.

Based on the Definition 5, for an information system based on the binary-valued system, it can then be easily represented as a soft set. Thus, we can make a one-to-one correspondence between $(F, E)$ over $U$ with $S=\left(U, A, V_{\{0,1\}}, f\right)$.

\section{E. Analysis of Reduction Techniques}

In the following section, we will highlight the achievements from techniques proposed [2]-[4]. As an illustration, let we consider the following case.

Example 1: Let a soft set $(F, E)$ represents studies on patients treated at a hospital. Let assume that there are 15 patients that are undergoing for gastric cancers treatment in the universe $U$ with $U=\left\{u_{1}, u_{2}, \cdots, u_{14}\right\}$, and the set of parameters that represent the symptoms and categories of gastric cancer as $E=\left\{p_{1}, p_{2}, p_{3}, p_{4}, p_{5}, p_{6}\right\}$. For the mapping of $F: E \rightarrow P(U)$ governed by "symptoms observed (.)", where (.) is to be filled in by one of the parameters $p \in E$. As an example, thus the overall approximation can be represented as in Fig. 1. 
$(F, E)=\left\{\begin{array}{c}\text { indigestio } n=\left\{\mathrm{u}_{1}, \mathrm{u}_{2}, \mathrm{u}_{3}, \mathrm{u}_{12}, \mathrm{u}_{13}\right\}, \\ \text { no appetite }=\left\{\mathrm{u}_{1}, \mathrm{u}_{2}, \mathrm{u}_{3}, \mathrm{u}_{12}, \mathrm{u}_{13}\right\}, \\ \text { abdominal discomfort }=\left\{\mathrm{u}_{2}, \mathrm{u}_{3}, \mathrm{u}_{12}, \mathrm{u}_{13}\right\}, \\ \text { fatique }=\left\{\mathrm{u}_{2}, \mathrm{u}_{13}, \mathrm{u}_{14}\right\}, \\ \text { bloating }=\left\{\mathrm{u}_{2}, \mathrm{u}_{4}, \mathrm{u}_{5}, \mathrm{u}_{6}, \mathrm{u}_{7}, \mathrm{u}_{8}, \mathrm{u}_{10}, \mathrm{u}_{11}, \mathrm{u}_{12}, \mathrm{u}_{13}\right\}, \\ \text { nausea }=\left\{\begin{array}{l}\mathrm{u}_{2}, \mathrm{u}_{3}, \mathrm{u}_{4}, \mathrm{u}_{5}, \mathrm{u}_{6}, \mathrm{u}_{7}, \mathrm{u}_{8}, \mathrm{u}_{10}, \mathrm{u}_{11}, \\ \mathrm{u}_{12}, \mathrm{u}_{13}, \mathrm{u}_{14}\end{array}\right\}\end{array}\right\}$

Fig. 1 The soft set

The previous example can be represented in the following Boolean-valued information system.

TABLE I

TABUlAR REPRESENTATION OF A SOFT SET FROM EXAMPLE 1

\begin{tabular}{|l|c|c|c|c|c|c|c|}
\hline$U / P$ & $p_{1}$ & $p_{2}$ & $p_{3}$ & $p_{4}$ & $p_{5}$ & $p_{6}$ & $f()$. \\
\hline$u_{1}$ & 1 & 1 & 0 & 0 & 0 & 0 & 2 \\
\hline$u_{2}$ & 1 & 1 & 1 & 1 & 1 & 1 & 6 \\
\hline$u_{3}$ & 1 & 1 & 1 & 0 & 0 & 1 & 4 \\
\hline$u_{4}$ & 0 & 0 & 0 & 0 & 1 & 1 & 2 \\
\hline$u_{5}$ & 0 & 0 & 0 & 0 & 1 & 1 & 2 \\
\hline$u_{6}$ & 0 & 0 & 0 & 0 & 1 & 1 & 2 \\
\hline$u_{7}$ & 0 & 0 & 0 & 0 & 1 & 1 & 2 \\
\hline$u_{8}$ & 0 & 0 & 0 & 0 & 1 & 1 & 2 \\
\hline$u_{9}$ & 0 & 0 & 0 & 0 & 0 & 0 & 0 \\
\hline$u_{10}$ & 0 & 0 & 0 & 0 & 1 & 1 & 2 \\
\hline$u_{11}$ & 0 & 0 & 0 & 0 & 1 & 1 & 2 \\
\hline$u_{12}$ & 1 & 1 & 1 & 1 & 1 & 1 & 6 \\
\hline$u_{13}$ & 1 & 1 & 1 & 1 & 1 & 1 & 6 \\
\hline$u_{14}$ & 0 & 0 & 0 & 1 & 0 & 1 & 2 \\
\hline$u_{15}$ & 0 & 0 & 0 & 0 & 0 & 0 & 0 \\
\hline
\end{tabular}

\section{F. Analysis of Attribute Reduction}

The concept of data reduction and decision making by means of the soft set theory was firstly proposed [2]. Firstly, it identified reduct soft sets using Pawlak's rough reduction. Then, one of the identified reduct soft sets is used for the decision making. The determination of the decision from the objects is based on the computed maximal weighted value.

One of the major drawbacks is data inconsistency since the identified reduct soft sets are not similar and thus will definitely yield different maximal weighted value from the identified reduct soft sets. Based on the proposed algorithm using Pawlak's rough reduction, it will allude to $U / P$ where $U=\left\{p_{1}, p_{2}, p_{3}, p_{4}, p_{5}, p_{6}\right\}$ as shown in the following example [2]

$$
\begin{gathered}
\frac{U}{P}=\left\{\left\{u_{1}\right\},\left\{u_{2}, u_{12}, u_{13}\right\},\left\{u_{4}, u_{5}, u_{6}, u_{7}, u_{8}, u_{10}, u_{11}\right\}\right. \\
\left.\left\{u_{9}, u_{15}\right\},\left\{u_{14}\right\}\right\}
\end{gathered}
$$

From such partition, the optimal objects will be $\left\{u_{2}, u_{12}, u_{13}\right\}$ as denoted by the maximum value of $6 .\left\{u_{3}\right\}$ has been identified as the sub-optimal. It has been defined that any subset of $E$ that will produce partition similar to the partition $U / P$ will be regarded as attribute reduction of $U$ [1]. The partition induced by $R \subset U$ is still

$$
\begin{gathered}
\frac{R}{P}=\left\{\left\{u_{1}\right\},\left\{u_{2}, u_{12}, u_{13}\right\},\left\{u_{4}, u_{5}, u_{6}, u_{7}, u_{8}, u_{10}, u_{11}\right\}\right. \\
\left.\left\{u_{9}, u_{15}\right\},\left\{u_{14}\right\}\right\}
\end{gathered}
$$

and an invariant to $U / P$ and therefore can be considered as an attribute reduct of $E$. Optimal objects are still $\left\{u_{2}, u_{12}, u_{14}\right\}$ denoted by the maximum value of 4 . While the values for the suboptimal object are $\left\{u_{3}, u_{4}, u_{5}, u_{6}, u_{7}, u_{8}, u_{10}, u_{11}, u_{14}\right\}$, which are different from suboptimal derived from $E$, i.e., $u_{3}$.

\section{G. Analysis of Attribute Parameter Reduction}

A new idea has been introduced to the process of decision making in soft set theory by introducing the concept of parameter reduction. The concept of parameterization reduction of soft sets is based on the situation such that $B \subset E$ is called a reduction of $E$ if $B$ is independent and $M_{B}=M_{E}$ [3].

For $f_{E}\left(h_{i}\right)=\sum_{j} h_{i j}$ where $h_{i j}$ are entered into the collection of $(F, E)$ and $M_{E}$ as the maximum value of $f_{E}$ represents the collection of objects in $U . A \subset E$ has been defined as a dispensable set, if $M_{E-A}=M_{E}$ [3]. In our example based on Example 1, we have obtained that $M_{E}=\left\{u_{2}, u_{12}, u_{13}\right\}$. Then, we let $S=\left\{p_{2}, p_{3}, p_{6}\right\}$ thus we have $M_{E-\left\{p_{2}, p_{3}, p_{6}\right\}}=\left\{u_{2}, u_{12}, u_{13}\right\}$ and $\left.M_{E-\left\{p_{2}, p_{3}, p_{6}\right.}\right\}=M_{E}$. Therefore, $S=\left\{p_{2}, p_{3}, p_{4}\right\}$, can be considered as a parameter reduction.

In [3] has succeeded in gaining consistencies for optimal object selection, but failed in upholding consistency for suboptimal objects. The choice for suboptimal objects is $\left\{u_{1}, u_{3}, u_{4}, u_{5}, u_{6}, u_{7}, u_{8}, u_{10}, u_{11}, u_{14}\right\}$, which were different from suboptimal derived from $E$ that is $\left\{u_{3}\right\}$.

\section{H. Analysis of Attribute Parameter Reduction}

The main objectives of normal parameter reduction are to provide consistency in selecting an optimal and suboptimal object for any set of reduct. It has maintained the same partitions of objects by defining that $f_{A}\left(u_{1}\right)=f_{A}\left(u_{2}\right)=\cdots=f_{A}\left(u_{n}\right)$ implies $C_{E}=C_{E-A}$ for the case of $A$ as dispensable [4].

In the case that for $A \subset E$, if $f_{A}\left(u_{1}\right)=f_{A}\left(u_{2}\right)=\ldots=f_{A}\left(u_{n}\right)$ implies $C_{E}=C_{E-A}$, then $A$ is called dispensable set. For this definition, it has used the normal parameter reduction to indicate $E-A$ [4]. The decision partition derived is as follow 


$$
\left\{\left\{u_{2}, u_{12}, u_{13}\right\},\left\{u_{3}\right\},\left\{u_{1}, u_{4}, u_{5}, u_{6}, u_{7}, u_{8}, u_{10}, u_{11}, u_{14}\right\}\right.
$$

and $\left\{u_{2}, u_{12}, u_{13}\right\}$ is the optimal objects and $\left\{u_{3}\right\}$ will be the sub-optimal objects. Let assume $Z=\left\{p_{3}\right\}, Z \subset E$. Since the decision partition generated after deleting $Z$ has not changed, that is $C_{E}=C_{E-Z}$. Therefore $Z$ is dispensable since. Thus, in [4] has successfully proven that the decision making is consistent when involved with the optimal and sub-optimal values. As can be seen in Table II, $\left\{u_{2}, u_{{ }_{12}}, u_{13}\right\}$ as the optimal objects and $\left\{u_{3}\right\}$ as the suboptimal object are still maintained.

TABLE II

TABULAR REPRESENTATION OF A SOFT SET FROM EXAMPLE 1

\begin{tabular}{|c|c|c|c|c|c|c|}
\hline$U / P$ & $p_{1}$ & $p_{2}$ & $p_{4}$ & $p_{6}$ & $p_{6}$ & $f()$. \\
\hline$u_{1}$ & 1 & 1 & 0 & 0 & 0 & 2 \\
\hline$u_{2}$ & 1 & 1 & 1 & 1 & 1 & 5 \\
\hline$u_{3}$ & 1 & 1 & 0 & 0 & 1 & 3 \\
\hline$u_{4}$ & 0 & 0 & 0 & 1 & 1 & 2 \\
\hline$u_{5}$ & 0 & 0 & 1 & 0 & 1 & 2 \\
\hline$u_{6}$ & 0 & 0 & 1 & 0 & 1 & 2 \\
\hline$u_{7}$ & 0 & 0 & 1 & 0 & 1 & 2 \\
\hline$u_{8}$ & 0 & 0 & 1 & 0 & 1 & 2 \\
\hline$u_{9}$ & 0 & 0 & 0 & 0 & 0 & 0 \\
\hline$u_{10}$ & 0 & 0 & 0 & 1 & 1 & 2 \\
\hline$u_{11}$ & 0 & 0 & 0 & 1 & 1 & 2 \\
\hline$u_{12}$ & 1 & 1 & 1 & 1 & 1 & 5 \\
\hline$u_{13}$ & 1 & 1 & 1 & 1 & 1 & 5 \\
\hline$u_{14}$ & 0 & 0 & 1 & 0 & 1 & 2 \\
\hline$u_{15}$ & 0 & 0 & 0 & 0 & 0 & 0 \\
\hline
\end{tabular}

\section{MATERIAL AND METHOD}

In this section, a new concept of object reduction based on hybrid reduction will be introduced. The main motivation for making this object reduction is to further reduce the size of the dataset, but at the same time is successful in upholding consistency.

Firstly, we define the notion of support for an object based on the co-occurrence of parameters on an object. Throughout the section, the pair $(F, E)$ refers to the soft set over the universe $U$ representing a Boolean-valued information system $S=\left(U, A, V_{\{0,1\}}, f\right)$. Let also assume that $U=\left\{u_{1}, u_{2}, \cdots, u_{j}\right\}$ and $E$ is a set of decision parameters, $E=\left\{e_{1}, e_{2} \ldots e_{n}\right\}$ where $j$ will represent the number of objects under consideration in the universe and $n$ will be the number of parameters characterizing the objects. By applying the concept of co-occurrence to an object in the Boolean-valued information based on soft set theory, we propose alternative techniques that are derived from the supported sets. The main objective of the proposed technique is to ensure that the process of parameter reduction when transforming the complex database into a much simpler database for decision making does not cause any changes to the hierarchies of the order of the supported set values from the objects. This is the most critical agenda when reducing the dataset as it ensures that the dataset remains consistent and accuracy has been maintained.

Definition 6: Let $(F, E)$ be a soft set over the universe $U$ and $u \in U$. A parameter co-occurrence set of an object $u$ can $\operatorname{coo}(u)=|\{e \in E: f(u, e)=1\}|$.

Based on Definition 6, the co-occurrence of an object will be based on the accumulative of the object's parameters.

Definition 7: Let $(F, E)$ be a soft set over the universe $U$ and $u \in U$. Support of an object $u$ is defined by $\operatorname{supp}(u)=|\{e \in E: f(u, e)=1\}|$.

The supported set as defined by Definition 7 defines supported set group membership. The supported set is a representation object of similar co-occurrences.

Definition 8: We can form ordered supported objects according to their support value as $U_{1}>U_{2}>\cdots>U_{3}$, where $U_{i} \subseteq U$.

Ordered supported ranked is important in this research as it will be used to determine the accuracy of the dataset as defined in Definition 8. Thus, $U_{i}$ is a collection of objects in $U$ having the same support i.e. objects of the same support are grouped into the same class. Obviously, $U=\cup_{1<i<n} U$ and $U_{i} \cap U_{j}=\varnothing$, for $i \neq i$. In other words, a collection of $U / E=\left\{U_{1}, U_{2}, \ldots, U_{n}\right\}$ is a decision of partition of $U$, socalled cluster decision of $U$.

The supported set is then ranked according to the supported set value. Thus, $U_{i}$ is a collection of objects in $U$ having the same support i.e. objects of the same support are grouped into the same class. In other words, a collection of $U / E=\left\{U_{1}, U_{2}, \ldots, U_{n}\right\}$ is a class decision partition of $U$, so-called cluster decision of $U$. All process of reduction would have to be referred to the class decision partition. Comparison of derived class decision partition, prior to the process of reduction and after the process of reduction will be made. If the decision class partition were similar, then the suggested set is a valid reduct set.

Definition 9: Let $(F, E)$ be a soft set over the universe $U$ and $u \in U$ and the support of an object $u$ is defined by $\operatorname{supp}(u)=\operatorname{card}(\{e \in E: f(u, e)=1\})$.

The supported set as defined by Definition 6 defines supported set group membership. The supported set is a representation object of similar co-occurrences. 
Definition 10: For soft set $(F, E)$ over the universe $U$ and $u \in U$. The ultimate maximum object is defined for an object $u$ which has the following condition $\operatorname{card}(\{e \in E: f(u, e)=1\})=|E|$ where $|E|$ is the cardinality of $E$.

The optimal object can be defined based on the amount of the cardinality of $E$.

Definition 11: For soft set $(F, E)$ over the universe $U$ and $u \in U$. An object $u$ will be the most optimal decision if $u$ has the ultimate maximum of $E$ and it is denoted by ultmax $(u)$.

If it equals to the maximum number of objects, then it qualifies to be categorised as an ultimate maximum object which eventually is the optimal object.

Definition 12: For soft set $(F, E)$ over the universe $U$ and $u \in U$. The ultimate minimum object is defined for an object $u$ which has the following condition $\operatorname{card}(\{e \in E: f(u, e)=0\})=|E|$.

The above Definition 9 states that if the cardinality count for an object is zero, then the object is known as a member of the ultimate minimum set. Thus, for any object of such stature, then it qualifies to be reduced from the dataset.

Definition 13: For soft set $(F, E)$ over the universe $U$ and $u \in U$. An object $u$ will be the most minimal decision if $u$ has the ultimate minimum of $E$ and it is denoted by ultmin $(u)$.

Based on Definition 11, any object that has been designated by the above definition is incapable of further reduction in its supported value. Therefore, it will be known as the ultimate minimum set. Any objects from the ultimate minimum set that is regarded as the most inferior object can be considered as dispensable and are known as part of the process of object reduction. The object that is reduced will form as an entity of inferior objects.

Definition 14: For soft set $(F, E)$ over the universe $U$, the least significant parameter is defined as $\operatorname{leastsig}(e)=\sum_{j} h_{i j}=0$.

Definition 15: Any parameter with the notion as least significant is an automated choice for to be dispensed.

Definition 16: For soft set $(F, E)$ over the universe $U$, the most significant parameter is defined as $\operatorname{mostsig}(e)=\sum_{j} h_{i j}=|E|$.

Based on Definition 14, any object that concurs to the definition of the ultimate maximum set will always be as identified the optimal object for the decision making. Since it is already established that for any object that is part of the ultimate maximum set as the optimal object, then it is permissible to partition it as an entity of optimal objects. By partitioning it to a separate entity, the objects that are dispensed from the original collection is part of the process known as objection reduction.

Definition 17: Any parameter with the notion as most significant is an automated choice for to be dispensed.

Parameter reduction based on its significance can be accomplished if it qualifies the description of either Definition 11 or Definition 13. By doing so, it will not affect the process of decision making due to the uniformity of the values in the objects. The following algorithm presents stepby-step in achieving significance based parameter reduction.

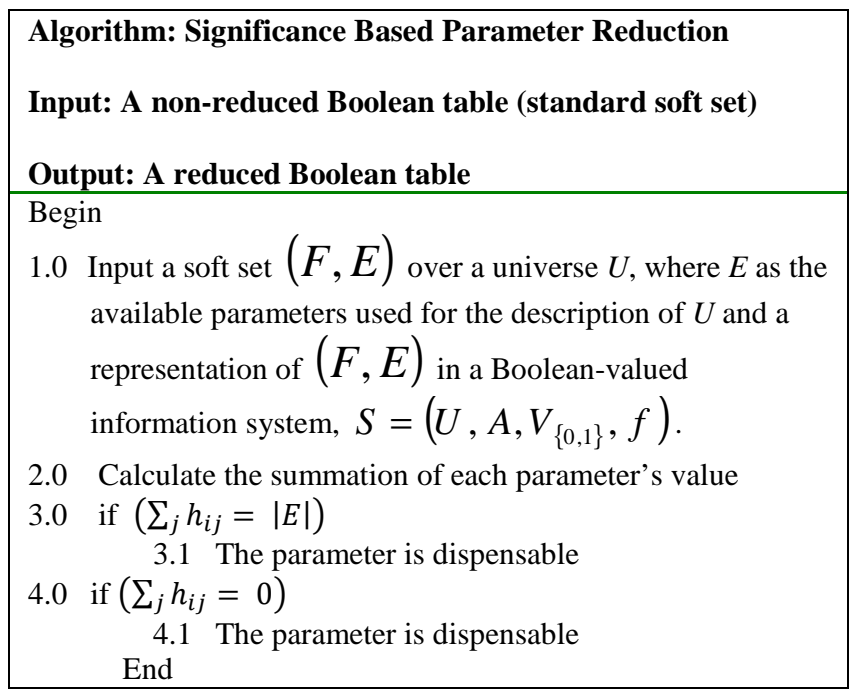

Fig. 2 The algorithm of the significance based parameter reduction

\section{RESULTS AND DISCUSSION}

Using Example 1 as our example and by referencing to [4], the decision partition formed is

$U / E=\left\{\begin{array}{l}\underbrace{\left\{u_{2}, u_{12}, u_{13}\right\}}_{\text {max }},\left\{u_{3}\right\}, \\ \left\{u_{1}, u_{4}, u_{5}, u_{6}, u_{7}, u_{8}, u_{10}, u_{11}, u_{14}\right\} \underbrace{\left\{u_{9}, u_{15}\right\}}_{\text {min }}\end{array}\right\}$ (5)

The parameter's support derived for each of the objects are as Fig. 3.

$$
\begin{aligned}
& \begin{array}{l}
\operatorname{Supp}\left(u_{i}\right)=6, i=2,12,13 \\
\operatorname{Supp}\left(u_{j}\right)=4, j=3 j=3 \\
\operatorname{Supp}\left(u_{k}\right)=2, k=1,4,5,6,7,8,10,11,14 \\
\operatorname{Supp}\left(u_{l}\right)=0, l=9,15 \\
\hline
\end{array} \text { Fig. 3 The support of each parameter }
\end{aligned}
$$

As can be seen from Fig. 1, we have identified the optimal objects are $\left\{u_{2}, u_{12}, u_{13}\right\}$. For these optimal objects, where $M_{E}=6$, they can be categorised as the ultimate maximum set. As in the above example, $\left\{u_{2}, u_{12}, u_{13}\right\}$ is the case of the ultimate maximum set because all the parameters 
are true. All the objects belonged to ultimate maximum support set are dispensable, and can be reduced by the process of object reduction. All objects that belong to ultimate maximum support set are partitioned into separate data set consisting only optimal objects of the ultimate maximum set. The concept of ultimate minimum sets can also be applied to $\left\{u_{9}, u_{15}\right\}$, since its support value is zero. We, therefore, have the new decision partition with the two entities as follow

$\mathrm{U} / \mathrm{E}=\left\{\begin{array}{l}\underbrace{\left\{\mathrm{u}_{2}, \mathrm{u}_{12}, \mathrm{u}_{13}\right.}_{\max }\},\left\{\mathrm{u}_{3}\right\}, \\ \{\mathrm{u}_{1}, \mathrm{u}_{4}, \mathrm{u}_{5}, \mathrm{u}_{6}, \mathrm{u}_{7}, \mathrm{u}_{8}, \mathrm{u}_{10}, \mathrm{u}_{11}, \mathrm{u}_{14} \underbrace{\hat{y}_{9}, \mathrm{u}_{15}}_{\text {min }}\}\end{array}\right\}$

By reducing the ultimate maximum and minimum, the newly formed decision partition is as follow:

$$
U / E=\left\{\left\{u_{3}\right\},\left\{u_{1}, u_{4}, u_{5}, u_{6}, u_{7}, u_{8}, u_{10}, u_{11}, u_{14}\right\}\right\}
$$

Let assume $Z=\left\{p_{3}\right\}$ and $Z \subset E$. If $Z$ is reduced from $E$, as observed the decision partition [4] is as follow

$$
U / E=\left\{\left\{u_{3}\right\},\left\{u_{1}, u_{4}, u_{5}, u_{6}, u_{7}, u_{8}, u_{10}, u_{11}, u_{14}\right\}\right\}
$$

By integrating the above-derived decision partition with the two ultimate entities, we have obtained the same decision partition

$\mathrm{U} / \mathrm{E}=\left\{\begin{array}{l}\underbrace{\left\{\mathrm{u}_{2}, \mathrm{u}_{12}, \mathrm{u}_{13}\right.}_{\max }\},\left\{\mathrm{u}_{3}\right\}, \\ \{\mathrm{u}_{1}, \mathrm{u}_{4}, \mathrm{u}_{5}, \mathrm{u}_{6}, \mathrm{u}_{7}, \mathrm{u}_{8}, \mathrm{u}_{10}, \mathrm{u}_{11}, \mathrm{u}_{14} \underbrace{\hat{y}_{\mathrm{u}_{9}, \mathrm{u}_{15}}^{\mathrm{u}_{1}}}_{\min }\}\end{array}\right\}$

Therefore, $\mathrm{Z}$ can be used as part of parameter reduction from the dataset. It has also been found that the data size has been reduced to $55 \%$ from the original size. Though data has been reduced by $45 \%$, the optimal, sub-optimal and inferior objects are consistently maintained.

\section{CONCLUSION}

As can be seen from our analysis [25], inconsistencies are the main issue that was neglected [2] as shown in the irregularities of the value of suboptimal objects. The major drawback is again inconsistency as shown in the selection [24] of suboptimal objects. The inferior objects have now become $\left\{u_{11}, u_{18}\right\}$ instead of $\left\{u_{7}\right\}$. To this, the problem of suboptimal choice and added parameter set of a soft set is analysed. With this technique, the optimal and suboptimal choices are still upheld. But, data has just been reduced by $17 \%$ from its original size [4].

\section{REFERENCES}

[1] D. Molodtsov, "Soft set theory-first results," Computers and Mathematics with Applications, vol. 37, pp. 19-31, Mar. 1999.

[2] P. K. Maji, A. R. Roy, and R. Biswas, "An application of soft sets in a decision making problem," Computer and Mathematics with Application, vol. 44, pp. 1077-1083, Nov. 2002.
[3] D. Chen, E. C. C. Tsang, D. S. Yeung, and X. Wang, "The parameterization reduction of soft sets and its applications," Computers and Mathematics with Applications, vol. 49, pp. 757-763, May 2005.

[4] Z. Kong, L. Gao, L. Wang, and S. Li, "The normal parameter reduction of soft sets and its algorithm," Computers and Mathematics with Applications, vol. 56, pp. 3029-3037, Dec. 2008.

[5] A. N. M. Rose, T. Herawan, and M. M. Deris, "A framework of decision making based on maximal supported sets," in Proc. ISNN'10, 2010, p. 473.

[6] F. Feng, Y. B. Jun, X. Liu, and L. Li, "An adjustable approach of fuzzy soft set based decision making," Computers and Mathematics with Applications, vol. 234, pp. 10-20, May 2010.

[7] Z. Kong, L. Gao, and L. Wang, "Comment on "A fuzzy soft set theoretic approach to decision making problems," Computers and Mathematics with Applications, vol. 223, pp. 540-542, Jan. 2009.

[8] Z. Kong, L. F. Wang, and Z. X. Wu, "Application of fuzzy soft set in decision making problems based on grey theory," Journal of Computational and Applied Mathematics, vol. 236, pp. 1521-1530, Oct. 2011.

[9] Y. C. Jiang, H. Liu, Y. Tang, and Q. M. Chen, "Semantic decision making using ontology-based soft sets," Mathematical and Computer Modelling, vol. 53, pp. 1140-1149, Mar. 2011.

[10] Y. C. Jiang, Y. Tang, and Q. M. Chen, "An adjustable approach to intuitionistic fuzzy soft sets based decision making," Applied Mathematical Modelling, vol. 35, pp. 824-836, Feb. 2011.

[11] X. Ma, H. Qin, N. Sulaiman, T. Herawan, and J. H. Abawajy, "The parameter reduction of the interval-valued fuzzy soft sets and its related algorithms," IEEE Transactions on Fuzzy Systems, vol. 22, pp. 57-71, Feb. 2014.

[12] W. Xu, Z. Xiao, X. Dang, D. L. Yang, and X. L. Yang, "Financial ratio selection for business failure prediction using soft set theory," Knowledge-Based Systems, vol. 63, pp. 59-67, Jun. 2014.

[13] Z. Xiao, K. Gong, and Y. Zou, "A combined forecasting approach based on fuzzy soft sets," Journal of Computational and Applied Mathematics, vol. 228, pp. 326-333, Jun. 2009.

[14] T. Herawan and M. M. Deris, "On multi-soft sets construction in information systems," in Proc. ICIC'09, 2009, p. 101.

[15] T. Herawan and M. M. Deris, "A soft set approach for association rules mining," Knowledge-Based Systems, vol. 24, pp. 186-195, Feb. 2011.

[16] A. N. M. Rose, T. Herawan, and M. M. Deris, "A framework of decision making based on maximal supported sets," in Proc. ISNN'10, 2010, p. 473.

[17] Y. Zao and Z. Xiao, "Data analysis approaches of soft sets under incomplete information," Knowledge-Based Systems, vol. 21, pp. 941-945, Dec. 2008.

[18] A. N. M. Rose, H. Hassan, M. I. Awang, N. A. Mahiddin, H. M. Amin, and M. M. Deris, "Solving incomplete datasets in soft set using supported sets and aggregate values," Procedia Computer Science, vol. 5, pp. 354-361, Dec. 2011.

[19] T. Deng and Y. Chen, "Comments from the author of "An objectparameter approach to predicting unknown data in incomplete fuzzy soft sets," Applied Mathematical Modelling, vol. 39, pp. 7744-7745, Dec. 2015.

[20] Zhao, F. Luo, S. K. M. Wong, and Y. Y. Yao, "A general definition of an attribute reduct," in Proc. ICRSKT'07, 2007, p. 101.

[21] T. Li, C. Luo, H. Chen, and J. Zhang, The Principles and Methodologies of Big Data Mining-From the Perspectives of Granular Computing and Rough Sets, Beijing, China: Science Press, 2016.

[22] C. C. Aggarwal, Data Mining: The Textbook, Berlin, Germany: Springer-Verlag, 2015.

[23] Z. Pawlak and A. Skowron, "Rudiments of rough sets," Information Sciences, vol. 177, pp. 3-27, Jan. 2007.

[24] I. M. Yassin, A. Zabidi, M. S. A. M. Ali, N. M. Tahir, H. A. Hassan, H. Z. Abidin, and Z. I. Rizman, "Binary particle swarm optimization structure selection of nonlinear autoregressive moving average with exogenous inputs (NARMAX) model of a flexible robot arm," International Journal on Advanced Science, Engineering and Information Technology, vol. 6, pp. 630-637, Oct. 2016.

[25] M. N. M. Nor, R. Jailani, N. M. Tahir, I. M. Yassin, Z. I. Rizman, and R. Hidayat, "EMG signals analysis of BF and RF muscles in autism spectrum disorder (ASD) during walking," International Journal on Advanced Science, Engineering and Information Technology, vol. 6, pp. 793-798, Oct. 2016. 